\title{
Biomicrofluidics: Recent trends and future challenges
}

\author{
TAMAL DAS ${ }^{\mathrm{a}}$ and SUMAN CHAKRABORTY ${ }^{\mathrm{b}, *}$ \\ a Department of Biotechnology, Indian Institute of Technology, Kharagpur 721302 \\ ${ }^{b}$ Department of Mechanical Engineering, Indian Institute of Technology, \\ Kharagpur 721302 \\ e-mail: suman@mech.iitkgp.ernet.in
}

\begin{abstract}
Biomicrofluidics is an active area of research at present, exploring the synergy of microfluidics with cellular and molecular biology, biotechnology, and biomedical engineering. The present article outlines the recent advancements in these areas, including the development of novel lab-on-a-chip based applications. Particular emphasis is given on the microfluidics-based handling of DNA, cells, and proteins, as well as fundamental microfluidic considerations for design of biomedical microdevices. Future directions of research on these topics are also discussed.
\end{abstract}

Keywords. Microfluidics; biomicrofluidics; lab-on-a-chip; micro total analysis system ( $\mu \mathrm{TAS})$.

\section{Introduction}

Ever increasing desire to explore biology at single entity level has made microfluidics remarkably paving into the biological domain. By virtue of its compatible length scale, explicit capability of exploring surface effects and unique ability to reduce the reagent volume, timescale and required manual labour, microfluidics has opened up novel and emerging vistas in the field of biological science, which, even a decade ago, looked utterly unattainable. Boosted by the recent exhilarating developments in the design and utilization of microfluidic devices for fluid transport, the technology of the micro Total Analysis Systems ( $\mu$-TAS) has revolutionized the processes associated with sample handling, detection and analysis in stand-alone integrated microfluidic platforms for bioassays. Applications of novel lab-on-a-chip devices range from bioscience industries for pharmaceuticals and medicine (e.g. drug design, detection and delivery, diagnostic devices) to industrial applications of combinatorial synthesis (e.g. high throughput screening and rapid chemical analyses). In conjunction, the ability to create fluidic structures mimicking biovasculation with micron-level precision in device length scales has given rise to new paradigms for non-invasive diagnostics and surgery with minimal post-surgical trauma. As an instance of the swiftly escalating demand for biomedical microdevices it should be acknowledged that the biochip market was $\$ 400 \mathrm{M}$ in the year 2000 and has subsequently grown eight-fold by 2006 (according to a market analysis by MarketReseach ${ }^{\mathrm{TM}}$ ). In the wake of such unprecedented advancement of microfluidics into

${ }^{*}$ For correspondence 
biomolecular and biomedical technologies, we have attempted to delineate a synoptic picture of the field's progress. Commencing with a concise discussion on the basic physics of microfluidics, we have included some major instances of application of microfluidics in cell biology, protein and DNA biophysics, structural biology, biosensors and finally tissue engineering.

\section{Microflow physics}

In order to design and optimize microfluidics-based devices for biological applications, it becomes essential to understand the underlying flow physics and interfacial phenomena at small scales. An excellent review of flow physics in micro- and nano-scale fluidic devices is presented in the review article by Squires \& Quake (2005). Here, we present a brief overview of the subject.

\subsection{Dimensionless numbers}

As it is evident from classical fluid mechanics, the flow physics may be grossly described by important dimensionless numbers delineating the interplay between different competing effects. For example, two of the most celebrated dimensionless numbers in the context of fluid mechanics and species transport are the Reynolds Number $(R e)$ and the solutal Peclet Number $(P e)$, depicting the ratios of inertial to viscous force and convective to diffusive force, respectively. Values of these parameters may belong to entirely different transport regimes altogether, as the system scale is reduced from macroscopic to a microscopic one.

Reynolds number, which is apparently the most important of all dimensionless numbers in forced flows, dictates the flow regime (laminar or turbulent) and is defined as the ratio of inertial to viscous force densities (Beebe et al 2002). In practice, Reynolds Number can be determined as

$$
R e=\rho v D_{h} / \mu,
$$

where $\rho$ is the density of the fluid, $\mathrm{v}$ is the characteristic velocity of the fluid, $D_{h}$ is the hydraulic diameter of the channel and $\mu$ is the viscosity of the fluid. In microflow regime where the flow velocity typically varies within $1 \mu \mathrm{m} / \mathrm{s}$ to $1 \mathrm{~cm} / \mathrm{s}$ and $D_{h}$ is of the order of $10 \mu \mathrm{m}$, the Reynolds number is obtained within $10^{-6}$ to 10 . In such a low Reynolds number governed flow regime, non-linearity due to the inertial effects is mostly eliminated and turbulence becomes unlikely. However, according to research studies reported by several groups, the value of the critical Reynolds number which signifies the transition from laminar to turbulent flow regime, has been observed to be remarkably less than the critical $R e$ for macro-scale flows (Mala $\&$ Li 1999). In order to explain these phenomena, investigations have been pursued in the direction of attributing the aforementioned deviation to the effects imparted by the surface features. In addition, an anomalous behaviour of flow friction for flows through microchannel has emerged as a major challenge to comprehensively understand the microfluidics flow physics (Weilin et al 2000). In a series of recent articles, the aforementioned anomalies have been explained by Chakraborty (2007a, b) and Chakraborty et al (2007c) on the basis of competing effects of stick effects induced by microchannel surface roughness and slip effects facilitated by nanobubble formation (figure 1). In this regard, even the laminar flow regime inside microfluidic network, which may be apparently perceived to be physically uninteresting 


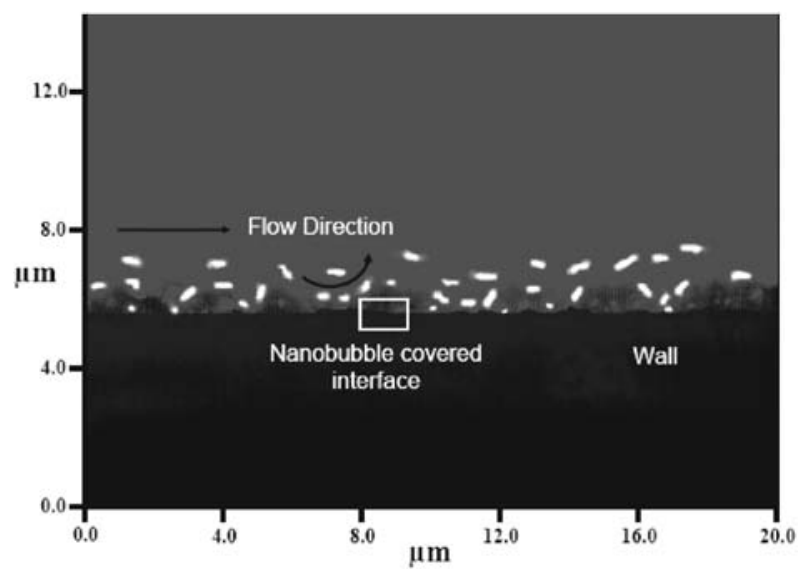

Figure 1. Imaging of typical near-wall fluid flow characteristics deciphering the hypothesized presence of nano-bubble.

(Chakraborty 2007b) as being devoid of the inertial non-linearities, definitely demands more fundamental attentions to be understood completely.

Owing to the low Reynolds number in microfluidic domain, mixing in microchannel is severely hindered. In disparity to macro scale flows where, for the most of cases, the origin of mixing can be traced to the chaotic eddies born out of the turbulence, mixing by diffusion is often the sole possible mechanism that can be realized in microchannels. However, depending on the targeted application, diffusive mixing can be either advantageous (e.g. reactions in microchannels) or harmful (e.g. particle separator). The dimensionless number that characterizes the nature and the strength of the diffusive mixing is called Peclet number $(\mathrm{Pe})$. By definition, Peclet number represents the relative strength of convection over diffusion, and is expressed as

$$
P e=v w / D,
$$

where $w$ is the width of the microchannel and $D$ is the diffusion co-efficient of the solute particles. Assuming $D$ to be of the order of $10^{-10} \mathrm{~m}^{2} / \mathrm{s}$ (which is true for most of the biomolecules), $w$ to be $10 \mu \mathrm{m}$ and $v$ to be $1 \mathrm{~mm} / \mathrm{s}$, it takes approximately 100 channels widths i.e. $1 \mathrm{~mm}$ in length before complete diffusive mixing occurs. In view of such weak strength of diffusiondependent mixing, different innovative strategies ascertaining mixing by secondary or transverse flow have been invented. Following theoretical predictions upheld by the works of Ajdari (2002), a staggered-herringbone structured chaotic micromixing device (Stroock et al 2002) has been fabricated, which considerably reduces the mixing length. In fact, there are ample instances where micromixers have been designed based on the mechanisms of - (i) passive mixing (Hardt et al 2005), such as Taylor dispersion (Huiqian et al 2006), hydrodynamic focusing (Veenstra et al 1999, Hessel et al 2003), split and recombine flows (Schönfeld et al 2004), flow separation (Mengeaud et al 2002), chaotic advection (Stroock et al 2002), DC electrokinetic instability (Oddy et al 2001) and surface charge patterning (Ajdari et al 1996; Erickson \& Li 2003), or, (ii) active mixing (Chang \& Yang 2007), such as rotation (Chou et al 2001), dielectrophoresis (Lee et al 2001), AC electrokinetic instability (Oddy et al 2001, Shin et al 2005), field-induced electroosmosis (Schasfoort et al 1999; Bazant \& Ben 2006) and electrowetting on di-electric (Lee et al 2002, Fair 2007). However, researchers are yet to accomplish an optimal strategy for mixing inside microchannels and thus, micromixing remains an area where new ideas can thrive opportunistically. 
Other dimensionless numbers that also become noteworthy in specific appliances are Knudsen number (Kn, signifies the ratio of molecular mean free path with the characteristic system length scale), Capillary number (Ca, ratio of viscous and surface tension forces), Weissenberg number (Wi, ratio of relaxation time and shear rate for polymers), and Deborah number (De, ratio of polymer relaxation time and characteristic flow time). Detailed discussions on these numbers can be found in the review article by Squires and Quake (2005) and are not repeated here for the sake of brevity.

\subsection{Developing surface effects}

As the surface area to volume ratio scales to the inverse of the device dimension, surface effects happen to be very prominent in microfluidics-based systems (Lee et al 2002; Huang et al 2003; Gascoyne \& Vykoukal 2004; Madou 2002). This has been typically explored in the generation of controlled monodisperse microdroplet between two immiscible fluids (Thorsen et al 2001) and the radius of the droplet has been shown scale as the inverse of Capillary number. As a matter of fact, in micron scale, the effective surface force is so pronounced over other competing forces that a stream of liquid can easily be confined, controlled or strategically driven by the judicious static or dynamic manipulation of solid-liquid or liquidliquid surface energies. This claim is certainly illustrated by the impressive bunch of emerging novel devices that relies on the dominant consequences imparted by the surface tension force. Pioneering example of this effect has been demonstrated by Zhao et al (2001), where the liquid has been confined within the desired domain and a 'virtual wall' has been created by selective patterning of surface energies of microchannel walls. With the physical background developed as above, we outline the following recent evolutions of microfluidic devices in the field of bioscience.

\section{DNA transport through microfluidics}

In earth life began with nucleic acid and specifically with the Deoxyribonucleic acid which (DNA) certainly plays a decisive role towards achieving the goal of fast disease detection and remediation. As such, DNA can be identified within the nucleus of each cell of every higher organism and has been proved to transmit the genetic information which ultimately, through the process of transcription (DNA to Ribonucleic acid i.e. RNa) and translation (RNA to protein) regulates the physiological processes of living organism. On structural basis, DNA is a linear polymer comprised of repeating nucleotide units that are covalently bonded together. The pieces of this sequence that contain the hereditary information are called genes. With accidental or evolutionary changes in the genetic information, collectively termed as mutation, genes stop performing appropriately resulting to physiological malfunctioning. Relevantly, for genetic ailments, patient's DNA can be analysed to detect any plausible possession of a mutation in a specific gene (Schena et al 1995, Brown \& Botstein 1999, Bier et al 1997). One of the various methods for achieving this purpose is to introduce multifarious different known single stranded short DNA sequences immobilized in a reactive microsystem. The DNA sample under investigation can be labelled with fluorescent dyes and 'hybridized' with these different sequences. Subsequently by identifying the specific DNA sequence with which this sample hybridizes, DNA sequence of the unknown sample can be determined accurately. This incidence can be attributed to the extraordinary high specific binding affinity of single stranded DNA sequences with exactly complementary single stranded sequences. It has been well recognized that this kind of hybridization of the DNAs to their complementary sequences 
plays a key role in central dogma for biological process comprising of replication, transcription and translation, where accurate recognition of nucleic acid sequences by their complementary strands is indispensable for the precise transmission of information content. With the intrinsic capability providing the rapid analysis of genetic information by hybridization, the DNA chips have gained extensive usage in bio-analytical chemistry, with emerging utilizations in important areas such as gene identification, genetic expression analysis, DNA sequencing and clinical diagnostics.

\subsection{DNA hybridization in microchannel}

A general principle of operation of the genetic mutation detection assays is to probe molecules that are immobilized to the solid substrates in order to detect the target DNA sequences in a given sample. Probe to target sequence binding is called passive hybridization if the binding event rely upon diffusion of target DNA molecules to the probe DNA molecules. It is very much pertinent to mention that most of the conventional DNA microarray devices principally depend on such passive nucleic acid hybridization which may take several hours. This is due to the fact in case of passive hybridization, the target DNA molecules, possessing diffusion coefficients typically of the order of $10^{-11} \mathrm{~m}^{2} / \mathrm{s}$, can only move close to the capture probes through a random Brownian motion prior to hybridization (Nkodo et al 2001). The aforementioned diffusion-dependence mechanism sets two major adverse effects on the way of achieving detectable hybridization signals and repeatable results - (i) large amounts of target DNA molecules are required for a complete assay and (ii) long time of incubation becomes essential. Consequently, efforts have been made to invent alternative methods that will expectedly result in a more fast and sensitive DNA sensing requiring low concentrations of target molecules. In this respect, pioneering technique was to exploit electric fields to enhance the rate of interaction between the probe and target DNA molecules accounting for the fact that the DNA strand contains uncompensated negatively charged groups. In diffusionbased transport of DNA, the time $(\tau)$ it takes a DNA molecule to travel over a distance $x$ is given as (Kassegne et al 2003)

$$
\tau=\frac{x^{2}}{2 D}
$$

where $D$ is the diffusion coefficient of the DNA molecule. However, in the case of electrophoresis, the time it takes to move a molecule in the electric field over a distance $x$, given by (Kassegne et al 2003)

$$
\tau=\frac{x}{\mu_{e p} E}
$$

where $\mu_{e p}$ is the electrophoretic mobility of the DNA and $E$ is the strength of the imposed electric field. Choosing typical values for $D\left(9.943 \times 10^{-11} \mathrm{~m}^{2} / \mathrm{s}\right)$ and $\mu_{e p}\left(15,000 \mu \mathrm{m}^{2} / \mathrm{V}-\mathrm{s}\right)$ and applied electric field of $0.004 \mathrm{~V} / \mu \mathrm{m}$, the electrophoretic transport may appear to be 150 times faster over a distance of $500 \mu \mathrm{m}$ (Heller et al 2000; Kassegne et al 2003). Even though fast DNA transport through the electrophoretic mode is certainly beneficial in many respects, one crucial drawback is that the sample solution containing the target DNA must be desalted prior to hybridization in order to eliminate any shielding of electric field by salt ions. The electric field depth being apparent only in close proximity to the electrodes in a solution with high salt-concentration, a high concentration of the ions eventually nullifies the 
electric field in the area away from the electrodes and diminishes the resultant electrophoretic mobility of the DNA molecules. Therefore, the rapid movement of DNA by an electric field imposes a low conductive buffer solution to be used, which stands very much in contrast to common molecular biology practices where high conductivity solutions are always preferred to achieve efficient hybridization. Subsequently, it becomes requisite to fabricate a DNA hybridization procedure that is capable of dealing with a broad range of buffer conductivities, and yet accomplish an optimal performance. Microfluidics-based DNA hybridization, in this respect, may play a pivotal role towards attaining this objective in the lab-on-a-chip format microdevices with enormous scientific importance.

In the recent past, microfluidics-based DNA hybridization strategies have been proposed and implemented to overcome the diffusion-controlled limits associated with passive DNA hybridization techniques (Fan et al 1999; Benoit et al 2001; Adey et al 2002; Wang et al 2003). There are numerous advantages coupled with such microfluidic DNA arrays: these facilitate the detection at the lowest possible DNA concentrations, allow for shorter timescale to achieve the desired level detection owing to the augmented mass transport, offer the capacity to examine several samples simultaneously in parallel by using a multichannel network approach, trim down the likely contamination by virtue of an enclosed apparatus, and secure integration of several functionalities in a single monolithic apparatus. A variety of flow-actuation mechanisms have been proposed by the researchers for achieving transport of the sample through the 'active' DNA hybridization chips. One of the common methods, known as electroosmotic (EO) pumping (Woolley et al 1996; Chiem \& Harrison 1997; Das et al 2006), fundamentally depends on the electroosmotic mechanism, which essentially indicates to the liquid flow by an externally applied electric field over charged liquid-solid interfaces. Fundamentally, electroosmosis can arise due to the formation of an electrical double layer (EDL) at the charged surfaces. As an effect of complex electro-chemical reactions, immobilized surface-charges can develop on a solid substrate which is in contact with an electrolyte. Consequently, the layer of ions adsorbed and immobilized on the charged surface in contact with an electrolyte is called the Stern layer. Due to counter-acting entropic and electrostatic interactions, the existence of such surface bound charges results in a re-distribution of neighbouring counter and co-ions in the bulk liquid phase. This leads to the development of an electric double layer, so that the local charge density in nearby regions of the interface becomes effectively non-zero. In the diffused layer of the EDL, the counter-ions dominate over the co-ions in order to balance the net surface charge. This diffuse part of the EDL extents over a spatial length scale away from the liquid-solid interface, the characteristic order of which being commonly recognized as the Debye length. Subsequently, the diffuse EDL tends to move due to electrostatic interactions if a potential is applied longitudinally along the microchannel axis and owing to the cohesive nature of the hydrogen bonding of the polar solvent molecules, the bulk buffer phase is effectively dragged along the microchannel axis, yielding into an electroosmotic flow (EOF). Such electroosmotic pumping systems function without additional movable mechanical parts, and thus enhance the long-term stability and diminish the difficulty of production. Furthermore, electroosmosis permits pumping of liquids over a spacious variety of conductivity, which is vital for most biochemical appliances. Also, from the implementation point of view, the integration of micro or nano scale metal electrodes in microfluidic systems is a comparatively straightforward procedure with the improvement of MEMS-based fabrication technologies. Therefore, the electrokinetic forces emerge to be perfect for manipulating DNA molecules and performing microfluidic operations in the relevant biochemical systems. However, the buffers used in DNA hybridization often include salts in high concentrations, which subsequently reduce the electroosmotic effects to a substantial degree. From this perspective, 
pumping using mechanical pressure (Kim et al 2006) might apparently contain some advantages over the electroosmotic approach in the sense that the former is independent to the variations in the salt concentration, $\mathrm{pH}$, and intrinsic macromolecular charges. Yet due to the enormous pumping power requirements, significant sample dispersion and the lack of accurate control linked with pressure-driven microflows, mechanical pumping alone could not evolve as one of the most favoured choices for driving fluid flow through microchannels for DNA hybridization applications. Also, because of the high back-pressures generated due to the inherent large flow resistances of mechanically-pumped microchannel flows, leakage avoidance may itself impose a difficult operational trouble. As a trade-off, researchers have recently projected (Das et al 2006) the employment of combined electroosmotic and pressure-driven transport mechanisms, for guiding DNA samples through microfluidics-based hybridization chips. Additionally, the researchers have well acknowledged the needs for comprehensive theoretical modelling and thorough simulation studies on microfluidics-based DNA transport in order to impose stringent controls over the hybridization performance of these kinds of DNA-microassays without going for too many tedious and expensive in situ experimental trials. This helps to obtain the optimal system parameters with the most favourable hybridization characteristics within the constraints of the chosen configuration.

\subsection{DNA separation in microchannel}

Another method to scrutinize the genetic aberration is to perform a gel based electrophoretic separation of nucleic acid fragments formed by specifically digesting a patient's DNA with restriction endonuclease. Dissimilarities in fragment lengths as obtained from the patient's DNA and a healthy reference individual indicate the possibilities of certain genetic ailments. This step can be followed by biochemical sequencing of the target fragments to acquire detailed knowledge about the nature of mutation. Disadvantages associated with conventional gel-electrophoresis based separation are high volume of sample and long time scales which are required for generating detectable and distinguishable signals. In addition, the conventional gel electrophoresis often fails to distinguish between two or more closely matching yet biochemically different sequences. Therefore, a continuous effort has been put forward by research communities to deduce a microfluidics-based device that will eventually overcome the aforementioned bottlenecks. One such pioneering attempt is the introduction of microfluidics enhanced capillary electrophoresis (CE) or capillary array electrophoresis (CAE) (Medintz et al 2001). In recent past, a number of CE and CAE devices have been fabricated based on the principle of electrokinetic flow inside microchannel, each containing different surface coating in order to gradually improve the functioning of the instrument (Loughran et al 2005). In a typical process, samples are injected into the device by integrated injectors such as. cross or double tee intersections (Jacobson et al 1994) while laser-induced fluorescence (LIF) has been the most established detection scheme (Liu \& Guttman 2004). Microfabricated capillary electrophoresis devices present multifarious advantages for genetic analysis including reduced sample volumes, higher speed, enhanced sensitivity and ability to perform parallel assays in single monolithic platform. In conjunction, design, fabrication and operation of capillary electrophoresis can be further simplified with a radial channel array format, leading to an even better high-throughput device for DNA separation (Medintz et al 2001). Rapid multiple allelic genotyping has become possible with the advent of such highperformance devices (Medintz et al 2001; Liu \& Guttman 2004; Mitnik et al 2001; Tian et al 2000; Liu \& Guttman 2004).

In addition to the aforementioned process for DNA separation, other methods such as employing nano-sized post arrays (Huang et al 2002), electric field flow fractionation (EFFF) 
(Chen \& Chauhan 2005) and entropic trap arrays (Streek et al 2004, Han \& Craighead 2000) have also been devised, which enable fast DNA separation in microfluidic frameworks. In case of static (Huang et al 2002) and dynamic nano-sized post arrays, the self assembled or microfabricated post arrays act as the controlled sieving matrix and the device performs with more efficacy than pulsed field gel electrophoresis (PFGE) whereas DNA fragments are separated with the aid of a lateral electric field in EFFF. Further, the development of entropic trap arrays has entailed a physically interesting case for DNA fragment separation. In the ground of bottlenecks imposed by CE method in separating long DNA fragments, entropic trap arrays have been microfabricated, which work excellently for the separation of long DNA fragments (Han \& Craighead 2000). In this method, DNA fragments are injected into a narrow microchannel with alternating deep regions and shallow constrictions. While the thickness of constriction region is smaller than the radius of gyration of the DNA molecules, deep region is made large enough to accommodate DNA molecules in their equilibrium shape. With electrokinetic forcing, the molecules become trapped in the most entropically favourable deep region, which is governed by the ratio of molecule's equilibrium size and the device dimensions. In contrast to the general idea of CE-based separation where short fragments move faster than long ones, entropic trap arrays yield counter-intuitive results. Here, large fragments move more rapidly than short ones since these are thermally more favourable to cross the entropic barrier while short fragments are retained within the deep region. In consequence, there exists a critical length $\left(x_{c}\right)$ below which fragments fail to cross the entropic barrier. This critical length is related to the applied electric field $(E)$ by the following relation:

$$
x_{c} \propto 1 / E .
$$

Subsequent to the fundamental scaling considerations, the basic principle and the device operation have been analysed extensively from experimental (Han \& Craighead 2000) as well as rigorous theoretical basis (Streek et al 2004) and the design of the entropic trap arrays has been optimized to perform with remarkable efficacy.

\section{Microfluidics-based proteomics}

Although not as frequent as in case of DNA, specialized microfluidic devices have been utilized to explore the biophysical dynamics of proteins in such an extent that could not have been imagined ever before. In this review, we segregate the subject of protein isolation, separation, crystallization, biochemical and biophysical characterization in broad sub-sections as presented below.

\subsection{Protein isolation and purification}

The ever expansive integration of analytical chemistry to the lab-on-a-chip devices is most evident in case of protein isolation, pre-concentration and analysis starting from the biological cell sources. The process chain being exhaustively time consuming and manual labour intensive in conventional macroscale processes, this has attracted immediate attentions of the research groups developing futuristic high-throughput microfluidic devices. The basis of protein separation and isolation is very much similar to the capillary electrophoresis method that has been employed to separate DNA fragments with variable size. Only the processes involved in proteomic assays are more complicated owing to the complex biochemical and biophysical nature of proteins in comparison with simple configurations of DNA. In a typical 
procedure, at first, cells are chemically lysed to release all the intracellular contents including the targeted protein molecules into the solution. In the harbingering attempt by Li \& Harrison (1997), an electrokinetic set up was devised to perform controlled manipulation and lysis of erythrocyte cells in seconds, which was at least 100 times faster than the conventional lysis time. The functionality of cell lysis apparatus has been further improved by Schilling et al (2002) where they have utilized an H-filter mediated cell lysis in diffusive intermediate zones between cell suspension and the lysis buffer. In proteomic protocols, the cell lysis process is immediately followed by pre-concentration of desired compounds which may be available in trace amount, by field amplified sample stacking (FASS) method in microfluidic framework (deMello \& Beard 2003; Jacobson \& Ramsey 1995). In FASS, the sample is prepared in a low conductivity buffer and is introduced into the microchannel alongside a running-buffer of comparatively higher conductivity. A voltage being applied, the resultant electric field strength turns out to be higher in the low-conductivity buffer than in the running buffer, resulting to an enhanced analyte velocity in the sample flow zone. However, in the interface, the analyte molecules stack, yielding a narrow separation band. Similar methods that have been used in this specific purpose are field amplified injection (Kutter et al 1998) and postinjection sample stacking technique, and miceller electrokinetic chromatography (MEKC) (Quirino \& Terabe 1998) and isotachophoresis (ITP) (Prest et al 1999). Furthermore, on course of downstream processing, pre-concentrated samples can be purified in nano-molar precision by biochip integrated photo-polymerized polyacrylamide gel (Herr \& Singh 2004), post-array based filtration (Andersson et al 2000), liquid/liquid extraction (Tokeshi et al 2000) and solid phase extraction (Kutter et al 2000). Very recently protein containing samples have been demonstrated to be pre-concentrated and analysed in Nanogap array by Junction Gap Breakdown method (Lee et al 2007). Isolation protein in purified format being unavoidable pre-requirement for any molecular biology analysis, the microfluidic implementation in this field demands more scientific attention than ever before.

\subsection{Structural biology inside microchannel}

Obtaining crystals of structurally and functionally vital proteins for detailed atomistic studies of protein configuration are one of the key challenges that stand in firm resistance in the path of deciphering the molecular codes of biological science. The process is extremely time-consuming requiring multiple reactions to be run simultaneously and in some cases, it is practically unfeasible. And in this very aspect, the microfluidics is anticipated to impart tremendous influence on projected progress of the field. Multifarious applications spanning the complete domain of structural biology suggests that microfluidic tools can be indispensable in this respect (Hansen \& Quake 2003). In spite of novel technological developments in crystallization techniques such as in application synchrotron radiation sources, model building (Ogata 1998; Dauter 2002) and phasing technique (Perrakis et al 1999), protein crystallization still remains an open question for molecular biologists, where the introduction of microfluidic technology has become inevitable. While the macroscale automated crystallization platform remains a 'state-of-the-art' technology, it does not provide any global solution to the fundamental problems of crystallography i.e. instability of the liquid/liquid interface. One of the most effective methods of crystallization, namely the free interface diffusion (FID) technique, continues to be inaccessible to the researchers working in this field. Microfluidicsbased protein crystallization reactors offer reproducible solution to the implementation of FID technique. Till date, small and large-scale microfluidic crystallization chips (Hansen et al 2002; Hansen \& Quake 2003) have been designed, involving microreactors of nanoliter volume. 


\subsection{Protein kinetics and thermodynamics}

Integrated microfluidic devices are capable of determining the enzyme kinetics with the unprecedented precision, requiring extremely low volume of the expensive enzyme solution and tremendously reducing the assay time (Beebe et al 2002). Enzyme kinetics in microfluidic domains has been investigated for several enzymes such as liver transaminase (Moser et al 1997) and $\beta$-galactosidase (Hadd et al 1997), protein kinase A (Cohen et al 1999), acetylcholinesterase (Hadd et al 1999), etc. However, on-chip investigation is not confined within the kinetic domain. Instead, it has found incredible applicability in the regime of macromolecular thermodynamics, as depicted by the numerous research reports related to combinatorial effects of temperature and concentration gradient (Holden \& Cremer 2005) on the multivalent protein-receptor binding (Holden \& Cremer 2005), temperature dependent macromolecular folding (Mao et al 2002) and protein displacement i.e. Vroman effect (Holden \& Cremer 2005).

\section{Cell biology and microfluidics}

The intrinsic ability of microfluidics to deliver chemical stimuli with the spatial precision comparable to sub-cellular dimensions has opened a new paradigm of cell biology with excellent capacity to address cellular problems at single cell level. With microfluidic devices it is possible to expose cells to the gradient streams of chemicals such as hormones, cytokines, growth factors or chemoattractants and measure the corresponding responses individually (El-Ali et al 2006). As the cell physiology, phenotype and functionalities are governed by the neighbouring chemical and physical conditions, microfluidics creates a whole new way of determining the fate of a cell in vitro. Pertinently, it is the motivation of integrating several time consuming assay steps into solitary monolithic micro-platform that has swept the initial impetus of fabricating microfluidic systems for cell culture related appliances (Dittrich \& Manz 2006). Again, while majority of microfluidics-based cell cultures devices are simply miniaturized versions of the conventional lab scale processes, build on the basis of low sample volume requirement and compatible scaling of biophysical forces (Squires \& Quake 2005), there are ample instances of microflow systems that have been designed to exploit hitherto unknown regimes of cell biology. It is therefore little undermining to consider microfluidic devices as small scale petri-plates, rather they should be appreciated as the latest weapons for biologists to unlock the inaccessible realms of cell biology.

It is the inability of macro scale cell culture system to accurately quantitate the cellcell, cell-analyte and cell-extracellular matrix (ECM) interactions which has prompted the development of microfluidic-based cell culture systems (Folch \& Toner 2000). In recent years, the integration of cell culture with microfluidic systems has resulted in multifunctional platforms for obtaining fundamental biolophysical and biochemical insights on cells and tissues, as well as for the development of cell-based sensors with biochemical, biomedical and environmental applications. Although most of the microscale applications concentrate on the short term responses by the cells confined within the microdomain, several essential cellular processes occur over longer time scales. For instance, cell-cell interaction, cell differentiation, and proliferation necessitate the development of a microfluidics-controlled long-term cell culture platform with a homeostasis microenvironment. With this view, long-term cell culture platforms (Balagadde et al 2005) have been manufactured with functionality of exposing cells to the gradient of analyte concentrations (Huang et al 2005). Typically, cells are restricted into the desired regions of microchannels by micropatterning the channel surface 
with ECM proteins (which promote cell adhesion), or by utilizing hydrodynamic trap arrays (Wheeler et al 2003) or dielectrophoretic trap arrays (Voldman et al 2002). Confined cells are then exposed to spatially (Jeon et al 2002) and temporally (Lin et al 2004) regulated chemical stimuli in order to perceive the nature of intra-cellular signal transduction that occurs in response. Another class of applications that microfluidic devices are targeted for is the implementation of micro-biomimetics and tissue-engineering where basic operations of organ or tissue are explored with the variation of neighbouring micro-environment (Andersson \& van den Berg 2004). In this context, sincere and continuing research endeavours with respect to the fibroblast, vascular (Borenstein et al 2002), bone (Holy et al 2003), neuron (Taylor et al 2003), cartilage (Peterson et al 2002), liver (Power et al 2002) and stem (Kim et al 2006) cell cultures are manifested by numerous research articles that have been published within a very short time duration. Although there has been an enormous progress of microfluidics in the field of cell biology, future researches should be directed to comprehend the adverse effects fluid shear on cells (Leclerc et al 2006) and the packaging of long term cell culture units into the portable microfluidic framework (El-Ali et al 2006).

\section{Microfluidics for medical devices}

\subsection{Drug delivery}

In recent years, microfluidics-based technologies have been applied in drug delivery devices in order to phenomenally improve the performance and the accuracy of such processes. Advanced photolithographic techniques that were earlier implemented effectively for the design and fabrication of complex shaped microfluidic devices have made their way to construct sophisticated drug delivery devices. Such devices efficiently retain the therapeutic activity of a drug, which may still be administered with cellular scale precision and minimal pain. One such example of advanced drug delivery device is a microneedle (McAllister et al 2003). Microfabrication has been utilized to produce microneedles, which are orders of magnitude smaller in diameter comparable to the commercially available needles for disposable syringes and can mediate painless and spatially localized delivery of pharmaceutically important molecules into targeted cells or tissues. Pertinently it may be mentioned that the research related to the application of microneedles for gene and drug delivery are qualitatively classified into three broad areas namely cellular delivery, local delivery and systemic delivery (Hilt \& Peppas 2005). These microfabricated needles are capable of minimizing the required dose of the drug, lessening the drug related side effects and reaching hitherto inaccessible parts of the body (Langer \& Peppas 2003). Not only to human or animal subjects, fabricated microneedles have been used to introduce membrane impermeable macromolecules such as large molecular weight drug molecules, proteins, peptides and oligonucleotides into specific cells in vitro in order to study the particular effect on individual cellular scales. Subsequently, microneedles have been applied to deliver bioactive peptides to neural cells (Chen \& Wise 1997), to deliver antirestenosis drugs into rabbit arteries (Reed et al 1998) and in transdermal drug delivery (McAllister et al 2003). In this respect, stable painless transdermal drug delivery presents a major challenge for researchers due to the resistance imposed by stratum corneum (SC), the outer 10-15 $\mu \mathrm{m}$ thick layer of skin. Although several innovative experimental (Davis et al 2004) as well as theoretical (Lv et al 2006) endeavours can be found in recently published literatures, an optimized solution is yet to be achieved. Functionality of modern microfluidics-based drug delivery systems has further been enhanced by incorporating micropumps, microvalves and feedback sensors into the device module (Madou 2002). 
These components act like controlled actuators that are essential for a sustained release of the sensitive therapeutic agents. An innovative addition to this progress is the introduction of selfregulated drug delivery microdevices whose working principle relies upon the biomolecular sensing by intelligent polymeric compounds (Madou 2002).

\subsection{Biosensors}

Among all of the microfluidics-based biosensing systems, cell-based systems are probably the most recognized and well-applied ones. These are utilized to scrutinize the changes in sensor cells brought by pathogens, pollutants, drugs or any other biomolecules (Bousse 1996, Pancrazio et al 1999). One of the pioneering examples of cell-based biosensors is the one developed by DeBusschere and Kovacs (2001). In this case, a highly integrated portable cellbased biosensor system has been tested with low concentration Nifedipine, a calcium channel blocker. The system consists of a complementary metal oxide semi-conductor (CMOS) chip as digital interface with recording electrodes. In conjunction, to maintain cells in the microfluidic culture chamber for sufficiently long period in controlled temperature environment, the chip has been coupled with heater electrodes as the temperature controller system. For cell-based biosensor applications, neural cells are of specific targets (Pearce et al 2005) owing to their ability to generate recordable signals. Among other type of biosensors, DNA or RNA-based sensing devices have found wide applications in medical diagnosis (Zaytseva et al 2005). Messenger RNA (mRNA)-based sensors have been utilized to detect pathogenic organisms such as Bacillus anthracis, pathogen responsible for anthrax (Baeumner et al 2004), Cryptosporidium parvum which causes water-borne cryptosporidiosis (Baeumner et al 2001), Dengue virus (Zaytseva et al 2004) and Escherichia coli (Baeumner et al 2003). Detection systems for most of the biosensors as discussed above are either optical such as directly tagged fluorophores, caged fluorophores and flourophores confined within liposomes, or electrically based with sensitive dependence on the changes in the impedance or voltage (Pancrazio et al 1999). However, the aforementioned techniques are limited by the capability of detecting trace amounts. Label free methods of detection (Blanco et al 2006), utilizing total internal reflection fluorescence microscopy (TIRFM) technology, Mach-Zehnder interferometer (MZI) and cantilever based biosensors (Shipper et al 1997) have also received tremendous research attentions. Challenges related to the detection limit, global applicability and long term sustenance still remain in the field of biosensors. Also for cell-based biosensors, number of cells and cell-cell interactions are definitely the issues which are to be scrutinized in future, if precise quantitation is intended (El-Ali et al 2006).

\subsection{Miniaturized biomedical devices}

The ultimate goal of $\mu$ TAS is to miniaturize the conventional instruments commonly used in biomedical technology into the scale of microfluidics. With the advent of advanced MEMS technology, it has become even more feasible than ever to incorporate all the functional components of a macro-scale instrument into the restricted spatial domains of microchannel system. One such instance is the microfluidic-based polymerase chain reaction (PCR) chip (Pal et al 2005), where all the components facilitating sample preparation, DNA strand denaturation, annealing and extension are fused within an apparently complex microfluidic network. Also, the speed of DNA sequencing has been astonishingly augmented by 384-channel microfluidic format DNA sequencer (El-Difrawy et al 2005). Another popular example of $\mu$ TAS is the miniaturized fluorescently activated cell sorter (FACS) device (Fu et al 2002) with an integrated pneumatically activated pumps and valves that select individual cells on the basis of 
their fluorescent properties or affinity to the surface immobilized specific receptors (Chang et al 2005). In drug screening related applications, scaled down version of patch-clamp (Wood et al 2004) devices and the microfluidic system that mimics physio-biochemical operations (Power et al 2002) of liver have already been commercialized. Microfluidic-based stable or rapidly switchable concentration gradient generators, capable of quantifying cellular chemotaxis while incubated with biochemical excitations, have also attracted the interests of several research groups (Walker et al 2005).

\section{Conclusions}

In the last couple of decades, microfluidics has traversed almost every corner of life sciences, with the promise of more efficient device performance with low sample volume requirement and fast response time. However, there are several issues such as compatibility of biological entities with microfluidic devices, stability of living organisms in the constrained environments of microchannels, and inherent challenges of handling complex molecules in narrow confinements, which need to be sorted out before this perceptibly nascent technology becomes developed enough to be picked up by the researchers in commonplace laboratory applications. Fundamental research on fluid mechanics and transport phenomena over small scales as well as technological advancements on fabrication, process control, and optical diagnostics may indeed play a key role towards achieving that feat.

\section{References}

Adey N B, Lei M, Howard M T, Jenson J D, Mayo D A, Butel D L, Coffin S C, Moyer T C, Hancock A M, Eisenhoffer G T, Dalley B K, Mcneely M R 2002 Gains in sensitivity with a device that mixes microarray hybridization solution in a 25-mu m-thick chamber. Anal. Chem. 74: 6413-6417

Ajdari A 1996 Generation of transverse fluid currents and forces by an electric field: electro-osmosis on charge-modulated and undulate surfaces. Phys. Rev. E. 53: 4996-5005

Ajdari A 2002 Transverse electrokinetic and microfluidic effects in micropatterned channels: Lubrication analysis for slab geometries. Phys. Rev. E. 65: 016301 (1-9)

Andersson H, van der Berg A 2004 Microfabrication and microfluidics for tissue engineering: state of the art and future opportunities. Lab Chip 4: 98-103

Andersson H, Wijngaart W, Enoksson P, Stemme G 2000 Micromachined Flow-through FilterChamber for Chemical Reactions on Beads. Sens. Actuators B67: 203-208

Baeumner A J, Cohen R N, Miksic V, Min J H 2003 RNA biosensor for the rapid detection of viable Escherichia coli in drinking water. Biosens. Bioelectron. 18: 405-413

Baeumner A J, Humiston M C, Montagna R A, Durst R A 2001 Detection of viable oocysts of Cryptosporidium parvum following nucleic acid sequence based amplification. Anal. Chem. 73: $1176-1180$

Baeumner A J, Leonard B, McElwee J, Montagna R A 2004 A rapid biosensor for viable B. anthracis spores. Anal. Bioanal. Chem. 380: 15-23

Balagadde F K, You L C, Hansen C L, Arnold F H, Quake S R 2005 Long-term monitoring of bacteria undergoing programmed population control in a microchemostat. Science 309: 137-140

Bazant M Z, Ben Y 2006 Theoretical prediction of fast 3D AC electro-osmotic pumps. Lab Chip 6: $1455-1461$

Beebe D J, Mensing G A, Walker G M 2002 Physics and applications of microfluidics in biology. Annu. Rev. Biomed. Eng. 4: 261-86

Benoit V, Steel A, Torres M, Lu Y Y, Yang H J, Cooper J 2001 Evaluation of three-dimensional microchannel glass biochips for multiplexed nucleic acid fluorescence hybridization assays. Anal. Chem. 73: 2412-2420 
Bier F F, Kleinjung F, Scheller F W 1997 Real time measurement of nucleic acid hybridization using evanescent wave sensors-steps towards the genosensor. Sens. Actuators B38: 78-82

Blanco F J, Agirregabiria M, Berganzo J, Mayora K, Elizalde J, Calle A, Dominguez C, Lechuga L M 2006 Microfluidic-optical integrated CMOS compatible devices for label-free biochemical sensing. J. Micromech. Microeng. 16: 1006-1016

Borenstein J, Terai H, King K, Weinberg E, Kaazempur-Mofrad M, Vacanti J 2002 Microfabrication technology for vascular tissue engineering. Biomed. Microdev. 4: 167-175

Bousse L 1996 Whole cell biosensors. Sens. Actuators B Chem. 34: 270-275

Brown P, Botstein D 1999 Exploring the new world of the genome with DNA microarrays. Nat. Genet. 21: $33-37$

Chakraborty S 2007a Order parameter modelling of fluid dynamics in narrow confinements subjected to hydrophobic interactions. Phys. Rev. Lett. 99: 094504 (1-4)

Chakraborty S 2007b Towards a generalized representation of surface effects on pressure-driven liquid flow in microchannels. Appl. Phys. Lett. 90: 034108 (1-3)

Chakraborty S, Das T, Chattoraj S 2007c A generalized model for probing frictional characteristics of pressure-driven liquid microflows. Unpublished

Chang C C, Yang R J 2007 Electrokinetic mixing in microfluidic systems. Microfluid. Nanofluid. 3: $501-525$

Chang W C, Lee L P, Liepmann D 2005 Biomimetic technique for adhesion-based collection and separation of cells in a microfluidic channel. Lab Chip 5: 64-73

Chen J, Wise K D 1997 A multichannel neural probe for selective chemical delivery at the cellular level. IEEE Trans. Biomed. Eng. 44: 760-769

Chen Z, Chauhan A 2005 DNA separation by EFFF in a microchannel. J. Colloid Interface Sci. 285: 834-844

Chiem N, Harrison D J 1997 Microchip-based capillary electrophoresis for immunoassays: analysis of monoclonal antibodies and theophylline. Anal. Chem. 69: 373-378

Chou H P, Unger M A, Quake S R 2001 A Microfabricated Rotary Pump. Biomed. Microdevices 3: 323-330

Cohen CB, Chin-Dixon E, Jeong S, Nikiforov T 1999 Amicrochip-based enzyme assay for protein kinase A. Anal. Biochem. 273: 89-97

Das S, Das T, Chakraborty S 2006 Modelling of coupled momentum, heat and solute Transport during DNA hybridization in a microchannel in presence of electro-osmotic effects and axial pressure gradients. Microfluid. Nanofluid. 2: 37-49

Dauter Z 2002 New approaches to high-throughput phasing. Curr. Opin. Struct. Biol. 12: 674-678

Davis S P, Landis B J, Adams Z H, Allen M G, Prausnitz M R 2004 Insertion of microneedles into skin: measurement and prediction of insertion force and needle fracture force. J. Biomech. 37: $1155-1163$

deMello A J, Beard N 2003 Dealing with 'real' samples: sample pre-treatment in microfluidic systems. Lab Chip 3: $11 \mathrm{~N}-19 \mathrm{~N}$

DeBusschere B D, Kovacs G T A 2001 Portable cell-based biosensor system using integrated CMOS cell-cartridges. Biosens. Bioelectron. 16: 543-556

Dittrich P S, Manz A 2006 Lab-on-a-chip: microfluidics in drug discovery. Nat. Rev. Drug Discov. 5: 210-218

El-Ali J, Sorger P K, Jensen K F 2006 Cells on chips. Nature 442: 403-411

El-Difrawy S A, Lam R, Aborn J H, Novotny M, Gismondi E A, Matsudaira P, Mckenna B K, O'Neil T, Streechon P, Ehrlich D J 2005 High throughput system for DNA sequencing 76: 074301 (1-7)

Erickson D, Li D 2003 Three-dimensional structure of electroosmotic flow over heterogeneous surfaces. J. Phys. Chem. 107: 12212-12220

Fair R B 2007 Digital microfluidics: is a true lab-on-a-chip possible? Microfluid. Nanofluid. 3 : 245-281

Fan Z H, Mangru S, Granzow R, Heaney P, Ho W, Dong Q, Kumar R 1999 Dynamic DNA hybridization on a chip using paramagnetic beads. Anal. Chem. 71: 4851-4859 
Folch A, Toner M 2000 Microengineering of cellular interactions. Annu. Rev. Biomed. Eng. 2: 227-256

Fu A Y, Chou H P, Spence C, Arnold F H, Quake S R 2002 An integrated microfabricated cell sorter. Anal. Chem. 74: 2451-2457

Gascoyne P R C, Vykoukal J V 2004 Dielectrophoresis-based sample handling in general-purpose programmable diagnostic instruments. Proc. IEEE 92: 22-42

Hadd A, Jacobson S, Ramsey J 1999 Microfluidic assays of acetylcholinesterase inhibitors. Anal. Chem. 71: 5206-5212

Hadd A, Raymond D, Halliwell J, Jacobson S, Ramsey J 1997 Microchip device for performing enzyme assays. Anal. Chem. 69: 3407-12

Han J, Craighead H G 2000 Separation of long DNA molecules in a microfabricated entropic trap array. Science 288: 1026-1029

Hansen C, Quake S R 2003 Microfluidics in structural biology: smaller, faster .. . better. Cur. Opin. Struct. Biol. 13: 538-544

Hansen C L, Skordalakes E, Berger J M, Quake S R 2002 A robust and scalable microfluidic metering method that allows protein crystal growth by free interface diffusion. Proc. Natl. Acad. Sci. USA 99: $16531-16536$

Hardt S, Drese K S, Hessel V, Schönfeld F 2005 Passive micromixers for applications in the microreactor and $\mu$ TAS fields. Microfluid. Nanofluid. 1: 108-118

Heller M J, Forster A H, Tu E 2000 Active microelectronic chip devices which utilize controlled electrophoretic fields for multiplex DNA hybridization and other genomic applications. Electrophoresis 21: $157-164$

Herr A E, Singh A K 2004 Photopolymerized cross-linked polyacrylamide gels for on-chip protein sizing. Anal. Chem. 76: 4727-4733

Hessel V, Hardt S, Lowe H, Schönfeld F 2003 Laminar mixing in different interdigital micromixers: I. Experimental characterization. AIChE J. 49: 566-577

Hilt J Z, Peppas N A 2005 Microfabricated drug delivery devices. Int. J. Pharmaceutics 306: 15-23

Holden M A, Cremer P S 2005 Microfluidic tools for studying the specific binding, adsorption, and displacement of proteins at interfaces. Annu. Rev. Phys. Chem. 56: 369-387

Holy C, Fialkov J, Davies J, Shoichet M 2003 Use of a biomimetic strategy to engineer bone. J. Biomed. Mater. Res. 65: 447-453

Huang P J, Lee P J, Sabounchi P, Lin R, Lee L P 2005 Continuous perfusion microfluidic cell culture array for high-throughput cell-based assays. Biotechnol. Bioeng. 89: 1-8

Huiqian Y, Nguyen N T, Huang X 2006 Micromixer Based On Taylor Dispersion. J. Phys. 34: 136-141

Huang L, Zhang Y, Liao J H, Xu L N, Gu N, Shen H J 2003 Micro-pattering by Wettability Control of $\mathrm{SiO}_{2}$ Surface with Ultraviolet Irradiation. Surf. Rev. Lett. 10: 569-570

Huang L R, Tegenfedlt J O, Kraeft J J, Sturm J C, Austin R H, Cox E C 2002 A DNA prism for high-speed continuous fractionation of large DNA molecules. Nat. Biotech. 20: 1048-1052

Jacobson S C, Hergenroder R, Koutny L B, Ramsey J M 1994 Open Channel Electrochromatography on a Microchip. Anal. Chem. 66: 2369-2373

Jacobson S C, Ramsey J M 1995 Microchip Electrophoresis with Sample Stacking. Electrophoresis 16: 481-486

Jeon N L, Baskaran H, Dertinger S K W, Whitesides G M, Water L V D, Toner M 2002 Neutrophil chemotaxis in linear and complex gradients of interleukin-8 formed in a microfabricated device. Nat. Biotech. 20: 826-830.

Kassegne S K, Resse H, Hodko D, Yang J M, Sarkar K, Smolko D, Swanson P, Raymond D E, Heller M J, Madou M J 2003 Numerical modelling of transport and accumulation of DNA on electronically active biochips. Sens. Actuators B94: 81-98

Kim J H S, Marafie A, Jia X Y, Zoval J V, Madou M J 2006 Characterization of DNA hybridization kinetics in a microfluidic flow channel. Sens. Actuators B113: 281-289

Kim L, Vahey M D, Lee H Y, Voldman J 2006 Microfluidic arrays for logarithmically perfused embryonic stem cell culture. Lab Chip 6: 394-406 
Kutter J P, Ramsey R S, Jacobson S C, Ramsey J M 1998 Determination of Metal Cations in Microchip Electrophoresis using On-Chip Complexation and Sample Stacking. J. Microcolumn Sep. 10: 313-319

Kutter J P, Jacobson S C, Ramsey J M 2000 Solid phase extraction on microfluidic devices. J. Microcolumn Sep. 12: 93-97

Langer R, Peppas N A 2003 Advances in biomaterials, drug delivery, and bionanotechnology. AIChE J. 49: 2990-3006

Leclerc E, David B, Griscom L, Lepioufle B, Fujii T, Layrolle P, Legallaisa C 2006 Study of osteoblastic cells in a microfluidic environment. Biomaterials 27: 586-595

Lee J, Moon H, Fowler J, Schoellhammer T, Kim C J 2002 Electrowetting and electrowetting-ondielectric for microscale liquid handling. Sens. Actuators A95: 259-268

Lee J H, Chung S, Kim S J, Han J 2007 Poly(dimethylsiloxane)-based protein preconcentration using a nanogap generated by junction gap breakdown. Anal. Chem. 79: 6868-6873

LeeY K, Deval J, Tabling P, Ho C M 2001 Chaotic mixing in electrokinetically and pressure driven microflows. In: Proc $14^{\text {th }}$ IEEE Workshop on MEMS, Interlaken, Switzerland, pp 483-486

Li P C H, Harrison D J 1997 Transport, manipulation, and reaction of biological cells on-chip using electrokinetic effects. Anal. Chem. 69: 1564-1568

Lin F, Saadi W, Rhee S W, Wang S-J, Mittal S, Jeon N L 2004 Generation of dynamic temporal and spatial concentration gradients using microfluidics devices. Lab Chip 4: 164-167

Liu S, Guttman A 2004 Electrophoresis microchips for DNA analysis. Trends Anal. Chem. 23: $422-431$

Loughran M, Cretich M, Chiari M, Suzuki H 2005 Separation of DNA in a versatile microchip. Sens. Actuators B107: 975-979

Lv Y-G, Liu J, Gao Y-H, Xu B 2006 Modelling of transdermal drug delivery with a microneedle array. J. Micromech. Microeng. 16: 2492-2501

Madou M J 2002 Fundamentals of Microfabrication, Second ed. CRC Press, Boca Raton

Mala G M, Li D 1999 Flow characteristics of water in microtubes. Int. J. Heat Fluid Flow 20: 142-148

Mao H B, Yang T L, Cremer P S 2002 A microfluidic device with a linear temperature gradient for parallel and combinatorial measurements. J. Am. Chem. Soc. 124: 4432-4435

McAllister D V, Wang P M, Davis S P, Park J H, Canatella P J, Allen M G, Prausnitz M R 2003 Microfabricated needles for transdermal delivery of macromolecules and nanoparticles: fabrication methods and transport studies. Proc. Natl Acad. Sci. 100: 13755-13760

Mitnik L, Novotny M, Felten C, Buonocore S, Koutny L, Schmalzing D 2001 Recent advances in DNA sequencing by capillary and microdevice electrophoresis. Electrophoresis 22: 4104-4117

Medintz I L, Paegel B M, Mathies R A 2001 Microfabricated capillary array electrophoresis DNA analysis systems. J. Chromatography A924: 265-270

Mengeaud V, Josserand J, Girault H H 2002 Mixing processes in a zigzag microchannel: finite element simulations and optical study. Anal. Chem. 74: 4279-4286

Moser I, Jobst G, Svasek P, Varahram M, Urban G 1997 Rapid liver enzyme assay with miniaturized liquid handling system comprising thin film biosensor array. Sens. Actuators B44: 377-80

Nkodo A E, Garnier J M, Tinland B, Ren H, Desruisseaux C, McCormick L C, Drouin G, Slater G W 2001 Diffusion coefficient of DNA molecules during free solution electrophoresis. Electrophoresis 22: $2424-2432$

Oddy M H, Santiago J G, Mikkelsen J C 2001 Electrokinetic instability micromixing. Anal. Chem. 73: 5822-5832

Ogata C M 1998 MAD phasing grows up. Nat. Struct. Biol. 5: 638-640

Pal R, Yang M, Lin R, Johnson B N, Srivastava N, Razzacki S Z, Chomistek K J, Heldsinger D C, Haque R M, Ugaz V M, Thwar P K, Chen Z, Alfano K, Yim M B, Krishnan M, Fuller A O, Larson R G, Burke D T, Burns M A 2005 An integrated microfluidic device for influenza and other genetic analyses. Lab Chip 5: 1024-1032

Pancrazio J J, Whelan J P, Borkholder D A, Ma W, Stenger D A 1999 Development and application of cell-based biosensors. Ann. Biomed. Eng. 27: 697-711 
Pearce T M, Wilson J A, Oakes S G, Chiu S Y, Williams J C 2005 Integrated microelectrode array and microfluidics for temperature clamp of sensory neurons in culture. Lab Chip 5: 97-101

Perrakis A, Morris R, Lamzin V S 1999 Automated protein model building combined with iterative structure refinement. Nat. Struct. Biol. 6: 458-463

Petersen E, Spencer R, McFarland E 2002 Microengineering neocartilage scaffolds. Biotech. Bioeng. 78: 802-805

Powers M J, Domansky K, Kaazempur-Mofrad M R, Kalezi A, Capitano A, Upadhyaya A, Kurzawski P, Wack K E, Stolz D B, Kamm R, Griffith L G 2002 A microfabricated array bioreactor for perfused 3D liver culture. Biotechnol. Bioeng. 78: 257-269

Prest J E, Baldock S J, Bektas N, Fielden P R, Treves Brown B J 1999 A single electrode conductivity detector for use in electrophoretic separation systems. J. Chromatogr. A836: 59-65

Quirino J P, Terabe S 1998 Exceeding 5000-fold concentration of dilute analytes in micellar electrokinetic chromatography. Science 285: 465-468

Reed M L, Clarence W, James K, Watkins S, Vorp D A, Nadeem A, Weiss L E, Rebello K, Mescher M, Smith A J C, Rosenblum W, Feldman M D 1998 Micromechanical devices for intravascular drug delivery. J. Pharm. Sci. 87: 1387-1394

Schasfoort R B M, Schlautmann S, Hendrikse J, VandenBerg A 1999 Field-effect flow control for microfabricated fluidic network. Science 286: 942-945

Schena M, Shalon D, Davis R W, Brown P O 1995 Quantitative monitoring of gene expression patterns with a complementary DNA microarray. Science 270: 467-470

Schilling E A, Kamholz A E, Yager P 2002 Cell lysis and protein extraction in a microfluidic device with detection by a fluorogenic enzyme assay. Anal. Chem. 74: 1798-1804

Schönfeld F, Hessel V, Hofmann C 2004 An optimized split-and-recombine micromixer with uniform 'chaotic' mixing. Lab Chip 4: 65-69

Shin S M, Kang I S, ChoY K 2005 Mixing enhancement by using electrokinetic instability under time-periodic electric field. J. Micromech. Microeng. 15: 455-462

Shipper E F, Brugman A M, Dominguez C, Lechuga L M, Kooyman R P H and Greve J 1997 The realization of an integrated Mach-Zehnder waveguide immunosensor in silicon technology. Sens. Actuators B40: 147-53

Squires T M, Quake S R 2005 Microfluidics: Fluid physics at the nanoliter scale. Rev. Mod. Phys. 77: 977-1026

Streek M, Schmid F, Duong T T, Ros A 2004 Mechanisms of DNA separation in entropic trap arrays: a Brownian dynamics simulation. J. Biotech. 112: 79-89

Stroock A D, Dertinger S K W, Ajdari A, Mezic I, Stone H A, Whitesides G M 2002 Chaotic mixer for microchannnels. Science 295: 647-651

Taylor A, Rhee S, Tu C, Cribbs D, Cotman C, Jeon N 2003 Microfluidic multicomponent device for neuroscience research. Langmuir 19: 1551-1556

Thorsen T R, Roberts W, Arnold F H, Quake S R 2001 Dynamic pattern formation in a vesiclegenerating microfluidic device. Phys. Rev. Lett. 86: 4163-4166

Tian H J, Jaquins-Gerstl A, Munro N, Trucco M, Brody L C, Landers J P 2000 Single-strand conformation polymorphism analysis by capillary and microchip electrophoresis: a fast, simple method for detection of common mutations in BRCA1 and BRCA2. Genomics 63: 25-34

Tokeshi M, Minagawa T, Kitamori T 2000 Integration of a microextraction system on a glass chip: Ion-pair solvent extraction on Fe(II) with 4,7-Diphenyl-1,10-phenanthrolinedisulfonic acid and Tri-n-octylmethylammonium chloride. Anal. Chem. 72: 1711-1714

Veenstra T T, Lammerink T S J, Elwenspoek M C, vandenBerg A 1999 Characterization method for a new diffusion mixer applicable in microflow injection analysis systems. J. Micromech. Microeng. 9: 199-202

Voldman J, Gray M L, Toner M, Schmidt M A 2002 A microfabrication-based dynamic array cytometer. Anal. Chem. 74: 3984-3990

Walker G M, Sai J, Richmond A, Stremler M, Chung C Y, Wikswo J P 2005 Effects of flow and diffusion on chemotaxis studies in a microfabricated gradient generator. Lab Chip 5: 611-618 
Wang Y, Vaidya B, Farquar H D, Stryjewski W, Hammer R P, Mccarley R L, Soper S A 2003 Microarrays assembled in microfluidic chips fabricated from poly(methyl methacrylate) for the detection of low-abundant DNA mutations. Anal. Chem. 75: 1130-1140

Weilin Q, Mala G M, Li D 2000 Pressure-driven water flows in trapezoidal silicon microchannels. Int. J. Heat Mass Transfer 43: 353-364

Wheeler A R, Throndset W R, Whelan R J, Leach A M, Zare R N, Liao Y H, Farrell K, Manger I D, Daridon A 2003 Microfluidic device for single-cell analysis. Anal. Chem. 75: 3581-3586

Wood C, Williams C, Waldron G J 2004 Patch clamping by numbers. Drug Discov. Today 9: 434-441

Woolley T, Hadley D, Landre P, deMello A J, Mathies R A, Northrup M A 1996 Functional integration of PCR amplification and capillary electrophoresis in a microfabricated DNA analysis device. Anal. Chem. 68: 4081-4086

Zaytseva N V, Goral V N, Montagna R A, Baeumner A J 2005 Development of a microfluidic biosensor module for pathogen detection. Lab Chip 5: 805-811

Zaytseva N V, Montagna R A, Lee E M, Baeumner A J 2004 Multi-analyte single-membrane biosensor for the serotype-specific detection of Dengue virus. Anal. Bioanal. Chem. 380: 46-53

Zhao B, Moore J S, Beebe D J 2001 Surface-directed liquid flow inside microchannels. Science 291: $1023-1026$ 